\title{
Integration of Supply Chain Planning with Time and Resource Constrained Project Scheduling Problems for Building's Thermal Renovation Projects
}

\author{
Shadan Gholizadeh-Tayyar ${ }^{1}$, Jacques Lamothe ${ }^{1}$, Lionel Dupont ${ }^{1}$ \\ ${ }^{1}$ Toulouse University, Ecole des mines d'Albi-Carmaux, Industrial engineering \\ department, 81000, Albi Cedex 09, France \\ \{shadan.gholizadeh_tayyar, jacques.lamothe, lionel.dupont\}@mines-albi.fr
}

\begin{abstract}
CRIBA is a project that aims at industrializing thermal renovation processes of buildings. It consists in designing and configuring make-to-order insulated panels that will be installed on the external facade of the buildings to meet the thermal renovation objectives. Our study provides an optimization model that comprehensively plans supply chain network, which delivers insulated panels to the building's worksites, and schedules renovation activity that should be executed at the worksites under the limited quantity of the resources' availability. In this context, integration of supply chain planning problem with resource constrained multi project scheduling problem and time constrained project scheduling is of interest in realizing the decision making tool.
\end{abstract}

Keywords: Supply chain planning, resource constrained multi project scheduling problem, time constrained multi project scheduling problem, mixed integer programming.

\section{Introduction}

Generally, resource constrained multi project scheduling problem (RCMPSP) is defined as extension of resource constrained project scheduling problem (RCPSP). In RCMPSP the decision makers deal with simultaneous planning of multiple projects that use a common pool of resources for scheduling of their activities whereas in RCPSP one single project is aimed at scheduling while it uses its own dedicated resources. According to [1], nearby $90 \%$ of on-going projects in worldwide are executed in a multi-projects environment. In both RCPSP and RCMPSPs two major types of the constraints are distinguishable: (a) precedency constraints and (b) resource satisfaction constraints [2]. In this context, the activities are forced to use the resources up to the amount that is available on the periods. A modified approach of this hypothesis is considered in Time Constrained Project Scheduling problem, TCPSP, that it supposes additional quantity of the resources has temporarily to be allocated in certain periods, [3]. 
Furthermore, regarding types of the resources used in projects, two main sorts could be stood out: Renewable and non-renewable ones [4].

From the viewpoint of renewable resources, we use the approach of TCPSPs. Herein; the availability of the renewable resources such as labor-works, cranes and trucks can increase by renting additional limited quantities on the periods that the demand for these resource types may exceed the available amount by the activities.

From the viewpoint of non-renewable resources, like the basic RCMPSP, consumption of the nonrenewable resources of the model cannot exceed the available quantity. In our case, this quantity is limited by the capacity of supply chain that produces and supplies the resource to the projects' worksites. In this context, our attention is drawn to consider chain of the organizations, which coordinate together, to feed the final required product (the insulated panels in our case) at the projects' worksites and to plan optimally the quantity of materials that should be flowed between the organizations to satisfy projects' worksites on time. Noticeably, such a challenge relates a typical Supply Chain Planning, SCP, problem to the multiple projects scheduling problems.

To the best of our knowledge, integration of supply chain planning problem with resource constrained multi project scheduling problem and time constrained project scheduling is not considered up to now in the literature.

In the rest of this paper, we review the literature. Then, we define use case of the study. The decision making tool and the results are presented in following. Conclusion and future researches will be discussed at the final section.

\section{Literature Review}

The literature in resource constrained multi projects scheduling dates back to the 1960s. However, RCMPSP has not studied as expensively as single project scheduling [5]. Dealing with multi project scheduling problems distinguishes two main approaches: 1- Single project approach, wherein all the projects are joined together to make a single super-project. Herein, a single critical path is regarded in the scheduling of the projects. 2- Multi projects approach, in which the parallel separated projects are treated simultaneously while they use the same restricted resources of the management company. Different separated critical paths can be recognized in planning of the projects in multi projects approach.

Considering the multi projects problems with single project approach leads the researchers to the literature of RCPSPs. Different extensions of single project scheduling are defined in the literature. As a variant of RCPSPs, TCPSP would remarkably be applicable on practical project scheduling. Therein, a pre-defined duedate is given for completion of the project but it may be too tight to achieve with the resources that are available initially. TCPSP considers an approach that a given project can get complete on time by adding certain additional amounts of the resources [3], [6] and [7]. In our study, this idea is utilized as well in order to make closer the completion time of the projects' activities to the expected dates that are regarded. In [6], the authors develop a decomposition method to deal with a TCPSP 
problem with adjacent resources. [7] provides a two stage heuristic that solves several instances of TCPSPs to optimality.

In order to discover more the other extensions of the single project planning problems, we refer the readers to the reviews [8] and [9] and textbook [3].

Besides the variants and extensions, from viewpoint of objective functions, resource constrained project scheduling problems are grouped according to different objectives: (i) Time- based objectives minimizes targets like completion time, earliness, tardiness and lateness [10]; (ii) Quality-based objectives in which maximization of the projects' quality is regarded [11]; (iii) Cost-based objectives where the objective function minimizes the total cost of projects like execution costs, material costs, inventory holding costs, costs related to tardiness or earliness of the project, [12] and [13], and the costs of adding supplementary resources in TCPSPs, [3]; (iv) Net present value objectives that they are dealt with when certain predefined cash flows happen in the time periods. In fact, net present value maximization of the projects reflexes the time value of money in project scheduling problems, [14]; and, (v) multi objective models that investigate two or more of the above-mentioned objectives simultaneously in one model. In [15] criteria such as time, cost, quality and whole robustness are considered to build the multi objective model for scheduling multiple projects. Based on the importance of different objectives, the proposed model is expected to generate scheduling alternatives. For that purpose, a cloud genetic algorithm is proposed.

Multi projects approach little drew the attention of the researchers, [16]. In [16], the authors justify this choice because (1) it is more realistic, (2) it presents great opportunity for improvement and (3) critically. Within their work, they consider a RCMPSP with two lateness objectives, projects' lateness and portfolio lateness. Set of the priority rules for planning the resources of the model are used by the authors. They distinct several situation, in which priority rules perform poorly. In [17] different resource availability levels are intoduced for the multiple projects. In [18], the preemption of the resources is considered for the multiprojects. [19] uses queuing theory for multi project planning.

Dealing with the multi projects planning in both single project approach, and multi projects approach, engages the researchers with NP-hard problems. In term of computation time of the models, treating with small size of these problems is reasonable by the commercial softwares. In the large size of the problems, more than 50 activities for scheduling [20], application of the heuristics and meta-heuristics reduce considerably the computational time.

From the viewpoint of mathematical modeling, generally two types of the formulation are regarded: discrete time formulation and continuous time formulation. In discrete models the start time of the activities takes integer values whereas in continuous it can take a non-integer value as well. [21] presents two different types of models for each of the mentioned formulations. The authors compare the proposed models with six literature models in 3240 benchmark instances. They define three criteria of good, optimal and feasible to study and rank the proposed models in each of the types. Our model is built based on the discrete time formulation. For the other formulation and developments, we refer the readers to [22]. 
The last part of the literature relates to the supply chain planning topic, which is considered in our study to plan the procurement of the nonrenewable resources of the problem. The decisions in supply chain planning are made in three levels of operational, tactical and strategic. In operational planning, decision makers try to provide a decisional framework that corresponds to very short periods of time, [23]. Control and procurement planning of necessary materials and short-term transport policy decisions correspond to this level of decision making. The tactical level in supply chain aims to consider the decision that associate with medium terms of planning. They can be considered in the decision making for planning the procurement of required materials, controlling the inventories, planning for production and distribution of the products, [24]. Major and fundamental decisions of network such as locating new facilities among the existing ones, selection of new technologies and selection between potential suppliers are taken into account in strategic level of decision making, [25].

\section{Problem Definition and Formulation}

\subsection{Use Case}

This work is a part of a research project, called CRIBA, (Construction et Rénovation Industrialisé Bois Acier). The project is defined in building sector in France to industrialize thermal renovation activities of the buildings. The business is determined to accomplish by designing and configuring make-to-order panels that will be installed on external facades of the buildings to insulate them and to reduce their energy consumption. The main composing elements of the panels are wooden frames, insulation material types, external coating product types and high-insulated carpentries. The carpentries are mailnly made from aluminum, PVC and wood. Each composing element of the panels are procured by corresponding suppliers/ manufacturers. The ready-to-use panels are shipped from the panels' manufacturing unit to different buildings' worksites. Several renewable resources such as laborworks, trucks and cranes should be present at the worksites to make progress on installing the panels. After installation, the former carpentries should be removed from the buildings. In order to respect the sustainability rules regarded in the environmental engagements, the wastes of the worksites are shipped to a recycling center. It is worthwhile to mention that the stocking the panels is not allowded at the thermal renovation worksites, Fig 1.

\subsection{Problem Definition}

Our study attemps to present a mid-term comprehensive decision making tool for CRIBA project that is provided by considering the decisions engaged in planning and 


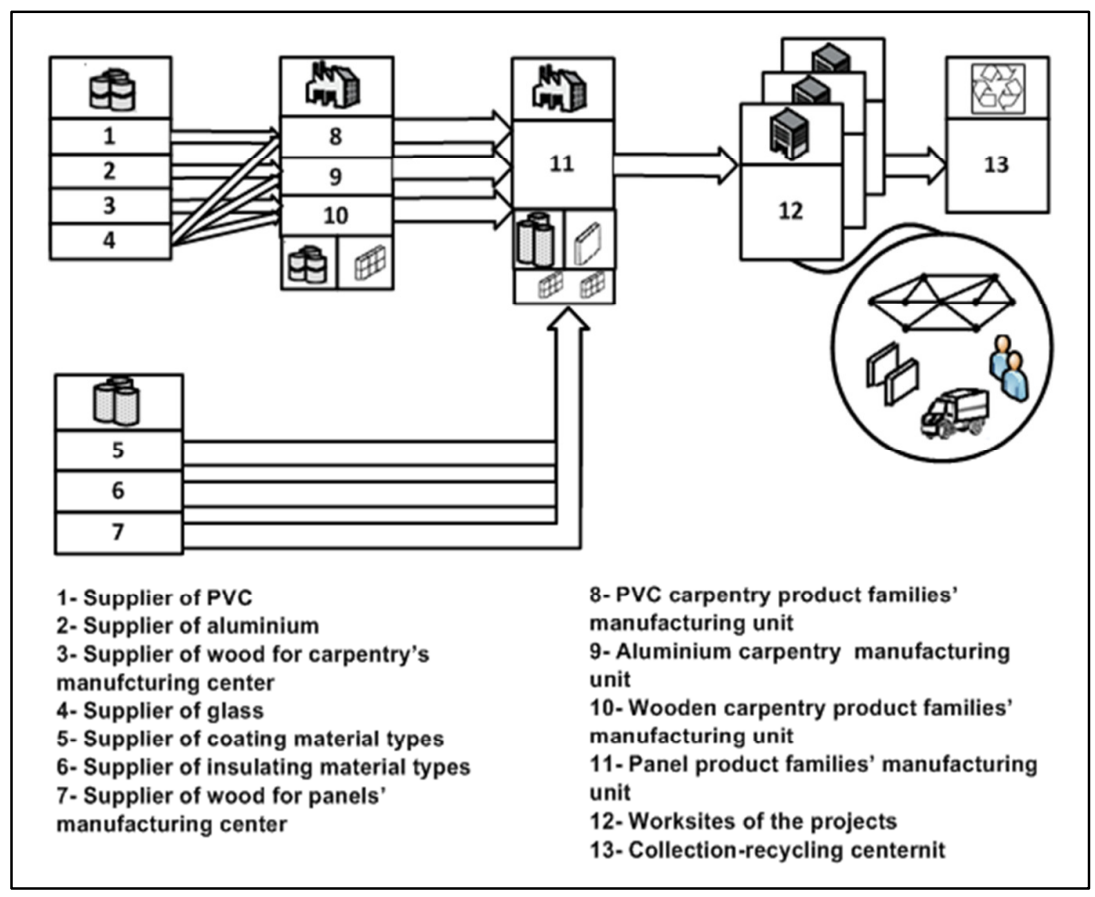

Fig. 1. Thermal renovation projects' framework.

scheduling of the projects' activities (project planning ) as well as the decisions faced on procuring the projects' required non renewable resources (supply chain planning) Following points represent our attributers for each of the approaches :

From the viewpoint of project planning : the model encompasses a set of $w$ projects which run at different buildings' worksites. Every project has its own specified activities, $\mathrm{I}_{\mathrm{w}}$. For each activity $i$, a processing time $\mathrm{Du}_{\mathrm{i}}$, earliest start time $\mathrm{e}_{\mathrm{i}}$, latest start time $l_{i}$, latest finish time $\mathrm{Lf}_{\mathrm{i}}$ and due-date $\mathrm{DD}_{\mathrm{i}}$ are attributed which are estimated by project managers. If the due date is not met, a penalty cost Penc $\mathrm{c}_{\mathrm{i}}$ will be imposed to the system. The predecessor relationship between two activities $i$ and $j$ is denoted by Pre $_{j}$. In addition, each activity uses certain amount of renewable resources, $\mathrm{Dr}_{\mathrm{ir}}$, and non-renewable resources, $\mathrm{Dp}_{\mathrm{ip}}$. The nonrenewable resources are supplied to the worksites with a limited capacity of its manufacturer over the different time periods. Meanwhile, $\mathrm{Ar}_{\mathrm{r}}$ units of renewable resource $r$ are available to share at the worksites. An additional quantity of renewable resource type $r$ can be rent with cost $\mathrm{Rc}_{\mathrm{r}}$. Relation set $\varphi_{1 \mathrm{w}}$ is defined to model a per period $\operatorname{cost} P c_{w}$ between the beginning and ending activity of the worksite $w$. 
From the viewpoint of supply chain planning: A network of organizations cooperate together to ultimately satisfy the demand of the worksites for the panels. One single organization is responsible to procure each type of the materials at the supply chain network. The set of the organizations $\mathrm{N}$ includes the carpentries' raw material suppliers $s_{c}$, the panels' raw material suppliers $s_{p}$, the manufacturing units of different carpentry $n_{c}$, the panels manufacturing unit $n_{p}$, worksites $w$ and wastes recycling center $r e,\left(\mathrm{~N}=\left\{s_{c}, s_{p}, n_{c}, n_{p}, w, r e\right\}\right)$. In the model, $o^{\prime}(n)$ indicates the articles that are consumed at the organization $n$, i.e. set of raw materials of the panels $r p$ and set of raw materials of the carpentries $r c$. Besides, $o(n)$ denotes the articles that are produced at the corresponding organization $n$. It can be panels $p$ or carpentries $c . N(o)$ is the destination units where a produced article $o$ can be used. In the supply chain network, in order to smooth the flow of products, each carpentry's and panel's manufacturing unit assigns a limited capacity to stock the required raw materials and the finished products, $\operatorname{Caps}_{n t}$. Beside of stocking the raw materials and ready-to-use panels, the panels' manufacturing unit stocks some limited quantity of the carpentries as well. $I S_{o^{\prime}(n), 0}$ and $I S_{o(n), 0}$ regard the initial stocks. Furthermore; Cappro $_{n t}$ and $\operatorname{Capsup}_{o^{\prime}(n), t}$ are defined to consider production capacity of the manufacturing centers and supply capacity of the organizations. Notation $P t_{o(n)}$ presents the workload of the production for producing the panels and carpentries at the corresponding manufacturing centers. In order to deal with the delays of the supply chain network, transportation lead-time $T l_{o(n), n} / T l_{o^{\prime}(n), n}$ and production lead-time $P t_{o(n)}$ are defined. Notation $B_{o, o^{\prime}}$ is generally used to define the bill of material of the product $o$ '.

Three different type of the costs including transportation costs $Q C_{o^{\prime}(n), n} / Q C_{o(n), n}$ production cost $P C_{o(n)}$ and stock costs $S C_{o^{\prime}(n)} / S C_{o(n)}$ are attended to calculate the total cost of the network.

\subsection{Mathematical Formulation}

Objective function: the objective of the model is to minimize total cost of the system execution. It includes different types of the costs:

- Project planning costs which includes total periodic cost of running the projects till their completion time, (ii) total penalty cost for lateness in completion of the jobs and (iii) total cost of renting the additional renewable resources.

- Supply chain planning cost including (v) the total transportation cost for shipping the raw materials and products at the network, (vi) production cost of the panels and carpentry types and (vi) the stock costs, which includes the stocking cost the raw materials and finished products at the carpentries' and panels' manufacturing units.

Min Z=

$$
\begin{aligned}
& {\left[\sum_{w} \sum_{(i, j) \in \varphi_{1 w}}\left(S_{j}+D u_{j}-S_{i}\right) P c+\sum_{i} \text { Ltns }_{i} \text { Penc }_{i}+\sum_{r} \sum_{t} R_{r t} R c_{r}\right]+} \\
& {\left[\sum_{o^{\prime}(n)} \sum_{n} \sum_{t} Q_{o^{\prime}(n), n, t} Q C_{o^{\prime}(n), n}+\sum_{o(n)} \sum_{n} \sum_{t} Q_{o(n), N(o(n)), t} Q C_{o(n), n}\right]+} \\
& {\left[\sum_{n \in\left\{n_{c}, n_{p}\right\}} \sum_{o(n)} \sum_{t} P Q_{o(n), n, t} P C_{o(n)}\right]+}
\end{aligned}
$$




$$
\left[\sum_{n \in\left\{n_{c}, n_{p}\right\}} \sum_{o^{\prime}(n)} \sum_{t} S_{o^{\prime}(n), n, t} S C_{o^{\prime}(n)}+\sum_{n \in\left\{n_{c}, n_{p}\right\}} \sum_{o(n)} \sum_{t} S_{o(n), n, t} S C_{o(n)}\right]
$$

\section{Constraints:}

\section{Supply chain design constraints}

Constraints (2) to (4) concern the capacity constraints. Constraints (2), (3) and (4) respectively deal with the production capacity, supply capacity and stock capacity of the organizations.

$$
\begin{array}{ll}
\sum_{o(n)} P t_{o(n), n} P Q_{o(n), n, t} \leq \text { Cappro }_{n, t} & \forall \mathrm{n} \in\left\{n_{c}, n_{p}\right\}, \mathrm{t} \in T \\
Q_{o^{\prime}, n, t} \leq \operatorname{Capsup}_{o^{\prime}, t} & \forall \mathrm{n} \in\left\{n_{c}, n_{p}\right\}, o^{\prime} \in O^{\prime}(n), \mathrm{t} \in T \\
\sum_{o^{\prime}(n)} S_{o^{\prime}(n), n, t}+\sum_{o(n)} S_{o(n), n, t} \leq \operatorname{Caps}_{n, t} \forall \mathrm{n} \in\left\{n_{c}, n_{p}\right\}, o^{\prime} \in O^{\prime}(n) \cup C, \mathrm{t} \in T
\end{array}
$$

Constraints (5)-(8) represent the balance of the flows. Equation (5) and (6) respectively consider the balance for stocked quantity of the finished products and the raw materials at their manufacturing centers considering their consumption, production and transportation. Equation (7) and (8) deal with the initial stocks.

$$
\begin{aligned}
S_{o, n, t-1}+P Q_{o, n, t-P l_{o}}=\sum_{N(o)} Q_{o, N(o), t}+ & S_{o, n, t} \\
& \forall \mathrm{n} \in n_{c} \cup n_{p}, o \in O(n), \mathrm{t} \in T \\
S_{o^{\prime}, n, t-1}+Q_{o^{\prime}, n, t-T l_{o^{\prime}, n}}=\sum_{o} P Q_{o, n, t} B_{o^{\prime}, o} & +S_{o^{\prime}, n, t} \\
& \forall o^{\prime} \in O^{\prime}(n) \cup C, \mathrm{n} \in n_{c} \cup n_{p}, \mathrm{t} \in T \\
S_{o^{\prime}, n, t}=I S_{o^{\prime}, n, 0} & \forall \mathrm{n} \in n_{c} \cup n_{p}, o^{\prime} \in O^{\prime}(n), \mathrm{t}=0 \\
S_{o(n), t}=I S_{o(n), 0} & \forall \mathrm{n} \in n_{c} \cup n_{p}, o \in O(n), \mathrm{t}=0
\end{aligned}
$$

Equation (9) presents the reverse logistic constraints. It guarantees that all the waste produced at the worksites will be shipped to the recycling center.

$$
Q_{o^{\prime}(r e), t}=\operatorname{Pro}_{o^{\prime}(w), w, t-T l_{o(r e)}} \quad \forall t \in T
$$

\section{Project planning constraints}

In this context, constraints (10)-(15) present the activities' scheduling constraints. Constraint (10) guarantees that all the activities of the projects should be executed in a time interval between their earliest start and latest start time. Equation (11) represents that the sum of the activity's execution periods should be equal with its processing time. Constraint (12) and (13) deal with the start time of the activities. Constraint (12) uses the activities' precedency relations for defining the start times. Equation (13) calculates the start time of the activities. Relying on the start times and activity's processing time, constraint (16) relates two decision variables $Z_{i t}$ and $U_{i t}$. Constraint (15) deals with the lateness may occur in executing the activities. It is considered by the difference between the due date, which is forecasted for completion of activity $i$, and the real completion time of activity $i$.

$$
\begin{array}{ll}
\sum_{t=e_{i}}^{l_{i}} Z_{i t}=1 & \forall i \epsilon I \\
\sum_{t=e_{i}}^{L F_{i}-1} U_{i t}=D u_{i} & \forall i \in I
\end{array}
$$




$$
\begin{array}{ll}
S_{j} \geq S_{i}+D u_{i} & \forall j \in \operatorname{Pre}_{j} \\
S_{i}=\sum_{t=e_{i}}^{l_{i}} t Z_{i t} & \forall i \epsilon I \\
\sum_{k=t}^{t-1+D u_{i}} U_{i k} \geq D u_{i} Z_{i t} & \forall i \in I, t \in\left\{e_{i}, \ldots, l_{i}\right\} \\
\text { Ltns }_{i} \geq S_{i}+D u_{i}-D D_{i} & \forall i \in I
\end{array}
$$

Constraints (16) and (17) deal with the resources availability constraints. Constraint (16) deals with the requirement of the activities for renewable resources. It guarantees that the demand for renewable resource types of the activities that are executing at a certain time period will be satisfied by the available quantities of these resources and the quantities that probably will be rented. Since panels cannot be stocked on the projects 'worksite, equation (18) guarantees that the total quantity of the panels that are transported to the worksites over a time period $t$ will satisfy the demand for the panels of activities that are progressing at time period $t$.

$$
\begin{array}{ll}
\sum_{i \in I_{n}} D r_{i r} U_{i t} \leq A r_{r}+R_{r t} & \forall r \in R, \mathrm{n} \in\{w\}, t \in T \\
\sum_{i \in I_{n}} Z_{i t} D p_{i, o^{\prime}(n)}=Q_{o^{\prime}(n), n, t} & \forall \mathrm{n} \in\{w\}, o^{\prime}(w) \in\{P\}, \mathrm{t} \in T
\end{array}
$$

Constraints (21), (22) and (23) represent the variable types that are used at modeling of the problem.

$$
\begin{array}{ll}
U_{i t}, Z_{i t} \in\{0 ; 1\} & \forall i \in I, t \in T \\
R_{r t}, L t n s_{i}, Q_{o(n), n, t}, P Q_{o(n), t}, & \forall o(n) \in O(n), r \in R, i \in I, \mathrm{n} \in N, t \in T(19) \\
S_{o(n), t}, S_{i} \in Z^{+} & \forall o^{\prime}(n), \in O^{\prime}(n), \mathrm{n} \in N, t \in T \\
Q_{o^{\prime}(n), n, t}, S_{o^{\prime}(n), t} \geq 0 &
\end{array}
$$

\subsubsection{Numerical Results}

Parameter setting presented in Table 1 is applied on the model. The problem is solved by using CPLEX algorithm on a notebook with Core i7 CPU, $2.7 \mathrm{GHz}$ processor, 64-bit and 4 GB of memory.

Table 1. Dimension of the indices of the model.

\begin{tabular}{lc}
\hline \multicolumn{2}{c}{ Indices } \\
\hline $\boldsymbol{i}:$ activities: $1, \ldots, 10$ & $\boldsymbol{r} \boldsymbol{p}$ : raw materials of the \\
$\boldsymbol{P}:$ panel types: $1,2,3$ & panels, \\
$\boldsymbol{W}:$ worksites: 1,2 & including: \\
$\boldsymbol{R}:$ renewable resource & insulating materials: \\
types: $1,2,3$ & $1,2,3$ \\
$\boldsymbol{t}:$ time periods: $1, \ldots, 10$ & Coating- \\
$\boldsymbol{C}:$ Product families of the & materials: $1,2,3$ \\
carpentries, & Wood materials:1,2,3 \\
including: & $\boldsymbol{r} \boldsymbol{c}:$ raw materials of the \\
PVC:1,2,3 & carpentries, $1, \ldots, 4$, \\
Wooden: $1,2,3$ & including: \\
Aluminum: $1,2,3$ & PVC, Aluminum, \\
& wood and glass. \\
\hline
\end{tabular}


Fig.2.A depicts the planning events that are engaged with each of the activitis, ( i .e. events related to start of the activies and also related to the procurement of the demanded consumable resources) Fig.2.B presents the total stock and production quantity of all the panel and carpentry types. Fig.2.C shows the graphs, which stand out the total quantity of the transported raw materials and products to the destination of use over the different time periods.

For the presented model, a failure in progressing of the thermal renovation activities over the time interval that are forecasted (execution of the activities between $e_{i}$ and $l_{i}$ ) originates from two kinds of reasons : one can be taken place by the failure of the supply chain network in supplying the resources and the other can be orginated from the worksites during the execution of the activities. In this study to deal with the effects of the failure in starting of the activities on the total cost of the system, we carry out sensitivity analyisis. For this purpose, we suppose that the earliest start time of the activities be postoponed for one unit of time. The results of the sensitivity analysis are presented inTable 2.

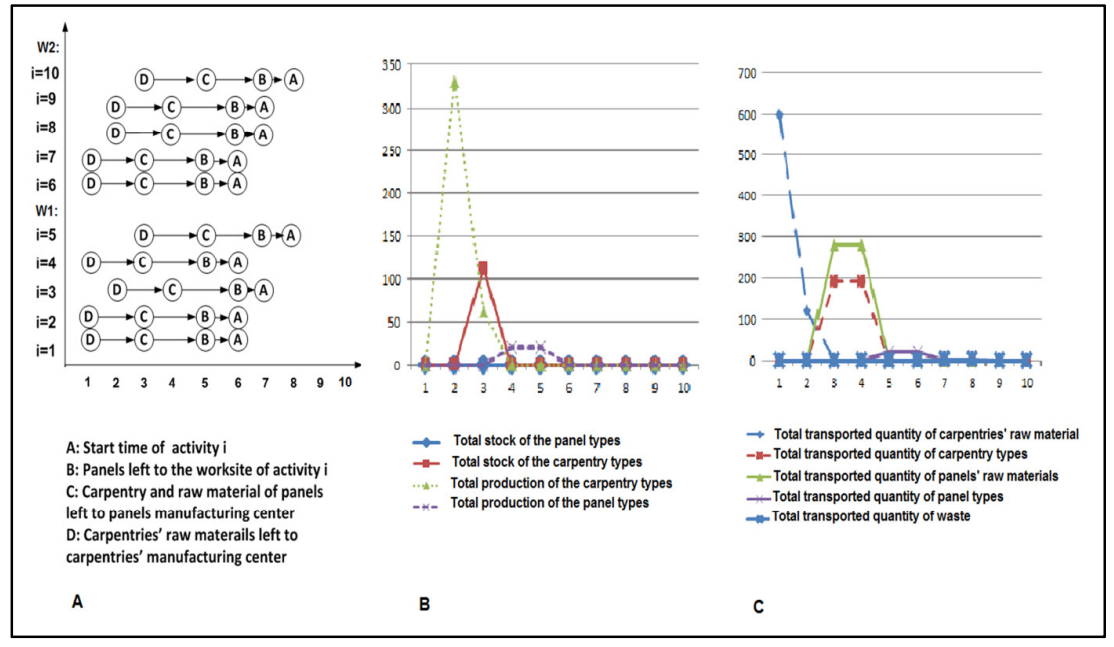

Fig.2. A :Start time of activities, B :total stock/ production quantities and C :total transported quantities of products and raw materials. 
Table 2. Results of the sensitivity analysis.

\begin{tabular}{l|ccc}
\hline Costivity & $\begin{array}{c}\text { Total Running } \\
\text { Cost in } \\
\text { Completion } \\
\text { Time,(i) }\end{array}$ & $\begin{array}{c}\text { Total Penalty } \\
\text { Cost, (ii) }\end{array}$ & $\begin{array}{c}\text { Total Resource- } \\
\text { add Cost, (iii) }\end{array}$ \\
\hline 1(in worksite1) & $0 \%$ & $3 \%$ & $0.23 \%$ \\
2(in worksite1) & $50 \%$ & $1 \%$ & $0.23 \%$ \\
3(in worksite1) & $50 \%$ & $1 \%$ & $0.23 \%$ \\
4(in worksite1) & $0 \%$ & $-1 \%$ & $0.23 \%$ \\
5(in worksite1) & $50 \%$ & $1 \%$ & $0.23 \%$ \\
6(in worksite2) & $0 \%$ & $3 \%$ & $0.23 \%$ \\
7(in worksite2) & $50 \%$ & $1 \%$ & $0.23 \%$ \\
8(in worksite2) & $50 \%$ & $1 \%$ & $0.23 \%$ \\
9(in worksite2) & $0 \%$ & $-1 \%$ & $0.23 \%$ \\
10(in worksite2) & $50 \%$ & $1 \%$ & $0.23 \%$ \\
\hline
\end{tabular}

\section{Conclusion and Future work}

In this study a multi project scheduling problem is regarded. Two types of the resources including renewable and nonrenewable resources are demanded by activities to do progress. A common pool of renewable resources with limited availability quantity is shared among the projects. In our model, we consider the possibility of renting additional quantity of the renewable resources, which lets the model to make closer the completion time of the activities, as much as possible, to the expected due dates (Integration of the time constrained project scheduling problems with multiple project scheduling problems). The non-renewable resources of the project are supplied at the worksites by their corresponding manufacturers. Procurement planning of these resources relates the model to the supply chain planning problem. The proposed optimization tool is built based on a case study that is defined by ADEME in context of CRIBA project. The results of solving the model for a small dimension of the problem are obtained by using CPLEX algorithm. A sensitivity analysis is carried out to study the effect of the failure in starting of the activities on different costs of the system.

For the future studies of this research, the authors aim at developing the model by applying the uncertainty modeling approaches such as robust optimization. Using the meta-heuristics to deal with large scale of the problem is also of interest.

\section{Acknowledgement}

The authors gratefully acknowledge the Agence d'Envirronnement et Maîtrise de l'Enegrie (ADEME) and SYRTHEA enterprise for founding of this research study. 


\section{References}

1. Payne, J.H.: Management of multiple simultaneous projects: a state-of-the-art review. International Journal of Project Management. 13, 163-168 (1995).

2. Badiru, A.B., Pulat, P.S.: Comprehensive Project Management: Integrating Optimization Models, Management Principles, and Computers. Prentice-Hall, Inc., Upper Saddle River, NJ, USA (1995).

3. Klein, R.: Schedulung of resource constrained projects. Spring Science \& Business (1999).

4. Shirzadeh Chaleshtarti, A., Shadrokh, S., Fathi, Y.: Branch and Bound Algorithms for Resource Constrained Project Scheduling Problem Subject to Nonrenewable Resources with Prescheduled Procurement. Mathematical Problems in Engineering. 2014, e634649 (2014).

5. Krüger, D., Scholl, A.: A heuristic solution framework for the resource constrained (multi-)project scheduling problem with sequence-dependent transfer times. European Journal of Operational Research. 197, 492-508 (2009).

6. Guldemond, T.A., Hurink, J.L., Paulus, J.J., Schutten, J.M.J.: Time-constrained project scheduling. Journal of Scheduling. 11, 137-148 (2008).

7. Hurink, J.L., Kok, A.L., Paulus, J.J., Schutten, J.M.J.: Time-constrained project scheduling with adjacent resources. Computers \& Operations Research. 38, 310-319 (2011).

8. Hartmann, S., Briskorn, D.: A survey of variants and extensions of the resourceconstrained project scheduling problem. European Journal of Operational Research. 207, $1-14(2010)$.

9. Węglarz, J., Józefowska, J., Mika, M., Waligóra, G.: Project scheduling with finite or infinite number of activity processing modes - A survey. European Journal of Operational Research. 208, 177-205 (2011).

10. Baker, K.R.: Minimizing earliness and tardiness costs in stochastic scheduling. European Journal of Operational Research. 236, 445-452 (2014).

11. Icmeli-Tukel, O., Rom, W.O.: Ensuring quality in resource constrained project scheduling. European Journal of Operational Research. 103, 483-496 (1997).

12. Ranjbar, M., Khalilzadeh, M., Kianfar, F., Etminani, K.: An optimal procedure for minimizing total weighted resource tardiness penalty costs in the resource-constrained project scheduling problem. Computers \& Industrial Engineering. 62, 264-270 (2012).

13. Rodrigues, S.B., Yamashita, D.S.: An exact algorithm for minimizing resource availability costs in project scheduling. European Journal of Operational Research. 206, 562-568 (2010).

14. Artigues, C., Demassey, S., Neron, E.: Resource-Constrained Project Scheduling: Models, Algorithms, Extensions and Applications. http://eu.wiley.com/WileyCDA/WileyTitle/productCd-1848210345.html (2008).

15. Browning, T.R., Yassine, A.A.: Resource-constrained multi-project scheduling: Priority rule performance revisited. International Journal of Production Economics. 126, 212-228 (2010).

16. Browning, T.R., Yassine, A.A.: Resource-constrained multi-project scheduling: Priority rule performance revisited. International Journal of Production Economics. 126, 212-228 (2010).

17. Dumond, E.J, Dumond, J.: An examination of resourcing policies for multi resource problems. International Journal of Operation \& Production Management. 13, 5478(1993).

18. Bock, D.B., Patterson, J.H.: A Comparison of Due Date Setting, Resource Assignment, 
and Job Preemption Heuristics for the Multiproject Scheduling Problem. Decision Sciences. 21, 387-402 (1990).

19. Navi-Isakow, S.,Golany.: Managing multiple project environments through constant work-in-process. International Journal of Project Management. 20, 127-130(2002).

20. Fatemi Ghomi, S.M.T., Ashjari, B.: A simulation model for multi-project resource allocation. International Journal of Project Management. 20, 127-130 (2002).

21. Kopanos, G.M., Kyriakidis, T.S., Georgiadis, M.C.: New continuous-time and discretetime mathematical formulations for resource-constrained project scheduling problems. Computers \& Chemical Engineering. 68, 96-106 (2014).

22. Hait, A., Artigues, C?: On electrical load tracking scheduling for a stel plant. Computers and Chemical Engineering. 68, 96-106 (2011).

23. Schütz, P., Tomasgard, A.: The impact of flexibility on operational supply chain planning. International Journal of Production Economics. 134, 300-311 (2011).

24. Chen, V.Y.X.: A 0-1 goal programming model for scheduling multiple maintenance projects at a copper mine. European Journal of Operational Research. 76, 176-191 (1994).

25. Chai, J., Liu, J.N.K., Ngai, E.W.T.: Application of decision-making techniques in supplier selection: A systematic review of literature. Expert Systems with Applications. 40, 3872-3885 (2013). 